\title{
Dominant components of the
} Thoroughbred metabolome characterised by $1 \mathrm{H}-\mathrm{NMR}$ spectroscopy: a metabolite atlas of common biofluids

Article

Accepted Version

Escalona, E. E., Leng, J., Dona, A. C., Merrifield, C. A., Holmes, E., Proudman, C. J. and Swann, J. R. (2015) Dominant components of the Thoroughbred metabolome characterised by $1 \mathrm{H}-\mathrm{NMR}$ spectroscopy: a metabolite atlas of common biofluids. Equine Veterinary Journal, 47 (6). pp. 721730. ISSN 2042-3306 doi: https://doi.org/10.1111/evj.12333 Available at https://centaur.reading.ac.uk/38453/

It is advisable to refer to the publisher's version if you intend to cite from the work. See Guidance on citing.

To link to this article DOI: http://dx.doi.org/10.1111/evj.12333

Publisher: Wiley

All outputs in CentAUR are protected by Intellectual Property Rights law, including copyright law. Copyright and IPR is retained by the creators or other copyright holders. Terms and conditions for use of this material are defined in the End User Agreement. 


\section{www.reading.ac.uk/centaur}

\section{CentAUR}

Central Archive at the University of Reading

Reading's research outputs online 


\section{Dominant components of the Thoroughbred metabolome characterised by ${ }^{1} \mathrm{H}-\mathrm{NMR}$ 2 spectroscopy: A metabolite atlas of common biofluids.}

3

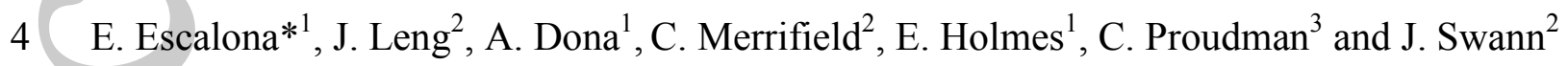
$5 \quad{ }^{1}$ Section Computational and Systems Medicine, Division of Surgery and Cancer, Faculty of 6 Medicine, Imperial College London, Sir Alexander Fleming Building, London SW7 2AZ, $7 \mathrm{UK}$;

$8{ }^{2}$ Department of Food and Nutritional Sciences, University of Reading, Whiteknights, 9 Reading, Berkshire, RG6 6AH, UK;

$10{ }^{3}$ Department of Gastroenterology/School of Veterinary Medicine, University of Liverpool, 11 Neston CH64 7TE, UK.

\section{2}

$13 *$ Corresponding author email: e.escalona10@imperial.ac.uk

14

15 Keywords: horse; metabonomics; metabolomics; metabolites; biofluids; nuclear magnetic

16 resonance

17

18

19 Summary

20 Reasons for performing study: Metabonomics is emerging as a powerful tool for disease 21 screening and investigating mammalian metabolism. This study aims to create a metabolic

This article has been accepted for publication and undergone full peer review but has not been through the copyediting, typesetting, pagination and proofreading process, which may lead to differences between this version and the Version of Record. Please cite this article as doi: 10.1111/evj.12333 
22 framework by producing a preliminary reference guide for the normal equine metabolic 23 milieu.

24 Objectives: To metabolically profile plasma, urine and faecal water from healthy racehorses

25 using high resolution ${ }^{1} \mathrm{H}-\mathrm{NMR}$ spectroscopy and to provide a list of dominant metabolites 26 present in each biofluid for the benefit of future research in this area.

27 Study design: This study was performed using seven Thoroughbreds in race training at a 28 single time-point. Urine and faecal samples were collected non-invasively and plasma was 29 obtained from samples taken for routine clinical chemistry purposes.

30 Methods: Biofluids were analysed using ${ }^{1} \mathrm{H}-\mathrm{NMR}$ spectroscopy. Metabolite assignment was

31 achieved via a range of $1 \mathrm{D}$ and $2 \mathrm{D}$ experiments.

32 Results: A total of 102 metabolites were assigned across the three biological matrices. A core 33 metabonome of 14 metabolites was ubiquitous across all biofluids. All biological matrices 34 provided a unique window on different aspects of systematic metabolism. Urine was the most 35 populated metabolite matrix with 65 identified metabolites, 39 of which were unique to this 36 biological compartment. A number of these were related to gut microbial host co37 metabolism. Faecal samples were the most metabolically variable between animals; acetate 38 was responsible for the majority (28\%) of this variation. Short chain fatty acids were the 39 predominant features identified within this biofluid by ${ }^{1} \mathrm{H}-\mathrm{NMR}$ spectroscopy.

40 Conclusions: Metabonomics provides a platform for investigating complex and dynamic 41 interactions between the host and its consortium of gut microbes and has the potential to 42 uncover markers for health and disease in a variety of biofluids. Inherent variation in faecal 43 extracts along with the relative abundance of microbial-mammalian metabolites in urine and 44 invasive nature of plasma sampling, infers that urine is the most appropriate biofluid for the 45 purposes of metabonomic analysis. 


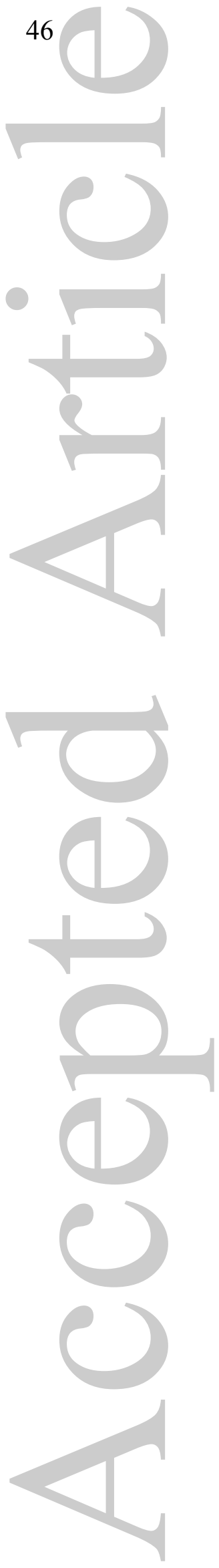

This article is protected by copyright. All rights reserved. 


\section{Introduction}

49 Metabonomics is a powerful systems biology approach that aims to simultaneously measure

50 all the low molecular weight metabolites present in a biofluid or tissue. This approach to 51 global untargeted characterisation of the metabolic phenotype allows the study of 52 multidimensional biochemical responses of complex biological systems to genetic or 53 environmental stimuli [1]. Metabolic profiling captures information from both intrinsic 54 (genetics, protein expression) and environmental inputs (diet, gut microbiota), providing 55 holistic information on the global system. This strategy has proven highly effective for

56 unravelling the complex metabolic interactions between the mammalian host and its resident

57 gut microbiota. Metabonomics is a tool of particular interest to equine researchers given the

58 vast impact of the equine gut microbiome on the bioavailability of food, medication and 59 energy. Metabonomics, along with other 'omic' technologies such as genomics, proteomics

60 and transcriptomics is increasingly showing potential in clinical settings as both a screening

61 tool and a means for mechanistic elucidation of disease pathways [2-4].

62 To date, there are fewer metabonomic studies exploring veterinary concerns than there are

63 human and rodent studies. The majority of mammalian work has concentrated on laboratory

64 animals [5-7] and animal models with high translatability to human health such as the pig [8;

$659]$. Less attention has been given to herbivorous hind-gut fermenters and the majority of

66 equine metabolic work concentrates on drug detection within the racing industry [10;11].

67 Equine-specific metabonomic studies include the use of the horse as a model for the

68 metabolic response to a dextrose challenge in type-2 diabetes [12] and a laminitic plasma

69 NMR study using an oligofructose overload model [13]. Other equine studies include 70 metabolic analysis of biofluids in response to age [14] and osteochondrosis [15]. Pappalardo 
71 et al. have explored metabolic variation in association with breed of horse and importantly

72 revealed significant differences that are likely to be attributed to differing growth rates and 73 protein utilisation [16]. These bodies of equine metabonomic work address the enormous 74 potential for exploring normal equine physiology and pathology-based variation. However, 75 there is still a paucity of baseline data on the metabolic phenotype of horses and this study 76 serves as a reference tool for the Thoroughbred racehorse for clinicians wishing to use 77 metabonomic technologies to complement their research in either a diagnostic or mechanistic 78 capacity.

79 Two major analytical platforms are commonly used for metabolic profiling, nuclear magnetic 80 resonance (NMR) spectroscopy and mass spectrometry (MS). These approaches

81 simultaneously capture quantitative information from a range of low molecular weight 82 metabolites across various different sample types. In metabonomic studies, NMR and MS are 83 used both independently and in tandem to achieve an in-depth coverage of the metabolome. 84 In this study we comprehensively characterise the dominant features within the urinary, 85 plasma and faecal metabolomes of Thoroughbred racehorses using ${ }^{1} \mathrm{H}-\mathrm{NMR}$ spectroscopy, as 86 this provides a reliable, reproducible [17] screening tool and is non-destructive of samples. 87 Thoroughbred racehorses represent a highly uniform equine population both genetically and 88 environmentally. Normal clinical chemistry parameters exist for this equine subtype to assist 89 racing industry standards and this study will build upon data already in the literature and in 90 clinical use.

91

92 Materials and methods

93

94 Sample collection 

From a large-scale study of urinary metabonomics of in-training Thoroughbred across a

96 number of yards, 7 animals were selected at random to provide a baseline metabolic reference 97 point for future comparison. Due to ethical constraints of invasive sampling and logistical 98 limitations of simultaneous collection of all 3 biofluids, 7 animals were selected as 99 representative of a wider population of animals. Early morning free-catch urine and faecal 100 samples were collected into sterile plastic containers and snap-frozen in liquid nitrogen 101 within 2 h. Plasma was obtained from excess clinical samples. Plasma samples were 102 collected into heparinised tubes, spun down to obtain the plasma fraction and snap-frozen 103 within $2 \mathrm{~h}$ of collection. All samples were then stored at $-80^{\circ} \mathrm{C}$ prior to $\mathrm{NMR}$ analysis.

104 Comprehensive metadata for each horse is shown in Supplementary Item 1 and samples were 105 consistently taken before a morning concentrate feed.

106

107 Sample preparation

108 Plasma and urine sample preparation was carried out as described by Beckonert et al. 109 2007[18].

110

111 Faecal samples $(100 \mathrm{mg})$ were combined with $1.7 \mathrm{~mm}$ Zirconia beads and $1 \mathrm{~mL}$ of distilled

112 water and homogenised in a bead-beater for $10 \mathrm{~min}$ and centrifuged at $13,000 \mathrm{~g}$ for $10 \mathrm{~min}$.

113 Water was evaporated from the samples using vacuum concentrator (Speed-Vac) and then 114 reconstituted in $700 \mu \mathrm{L}$ phosphate buffer $\left(\mathrm{pH} 7.4 ; 100 \% \mathrm{D}_{2} \mathrm{O}\right)$ containing $1 \mathrm{mM}$ sodium 3115 trimethylsilyl-1-[2,2,3,3-2H4]propionate (TSP).

116

117 Combined sampling approach for 2D NMR Experiments

118 2D spectra were obtained from pooled samples for each sample type to ensure comprehensive 119 capture of metabolites. $50 \mu \mathrm{L}$ of urine was pooled from each horse before the addition of 500 
$120 \mu \mathrm{L}$ of phosphate buffer (and $500 \mu \mathrm{L}$ of the resulting volume was added to the $5 \mathrm{~mm}$ NMR

121 tube). The same process was repeated for the plasma samples with the addition of $500 \mu \mathrm{L}$ of

$122 \mathrm{D}_{2} \mathrm{O}$ saline solution. For the faecal samples, individual samples were processed as previously

123 described and then pooled after reconstitution with $100 \mu \mathrm{L}$ of phosphate buffer.

124 Acquisition of $1 \mathrm{D}{ }^{1} \mathrm{H}-\mathrm{NMR}$ spectra

125 Spectroscopic analysis of all samples was carried out on a $700 \mathrm{MHz}$ Bruker NMR

126 spectrometer ${ }^{\mathrm{b}}$ operating at $300 \mathrm{~K}$ and equipped with a $5 \mathrm{~mm}{ }^{1} \mathrm{H}\left({ }^{13} \mathrm{C} /{ }^{15} \mathrm{~N}\right)$ inverse cryoprobe.

127 Urine and faecal samples

128 Standard one-dimensional ${ }^{1} \mathrm{H}-\mathrm{NMR}$ spectra were acquired for all urine and faecal samples.

129 We employed a standard one-dimensional pulse sequence (noesypr1d) that employs the first

130 increment of a NOE sequence to achieve suppression of the water resonance with water peak

131 suppression using a standard pulse sequence [19]. For each sample, 8 dummy transients were

132 followed by 256 transients and collected in $64 \mathrm{~K}$ data point. Irradiation of the solvent $\left(\mathrm{D}_{2} \mathrm{O}\right)$

133 resonance was applied during presaturation delay $(2.0 \mathrm{~s})$ for all spectra. The pulse sequence

134 parameters including the $90^{\circ}$ pulse, receiver gain and pulse powers were optimised for each

135 sample set run. The spectral width was $20 \mathrm{ppm}$ for all spectra. The free induction decay (FID)

136 was processed with an exponential line broadening of $0.5 \mathrm{~Hz}$ prior to Fourier transformation.

137 Plasma samples

138 Water-suppressed Carr-Purcell-Meiboom-Gill (CPMG) spin-echo spectra were acquired for

139 the plasma samples, Here, 8 dummy scans followed by 256 scans were acquired for each

140 sample in $64 \mathrm{k}$ data points with a total spin-spin relaxation delay of $1.5 \mathrm{~s}$ and a total delay

141 between pulse cycles of $4.85 \mathrm{~s}$.

142 Acquisition of $2 \mathrm{D}{ }^{1} \mathrm{H}-\mathrm{NMR}$ spectroscopy was undertaken with an $800 \mathrm{MHz}$ Bruker NMR

143 spectrometer $^{\mathrm{b}}$ operating at $300 \mathrm{~K}$ and equipped with a triple-resonance probe (TXI). J- 
144 resolved spectroscopy (J-res) spectra were acquired from all biofluid composite samples

145 using 64 transients per increment with 160 increments in the second dimension. The F1 (J146 coupling) domain spectral width covered $120 \mathrm{~Hz}$. Prior to the double FT and magnitude 147 calculation, the F1 data was zero-filled to 1024 points. The spectra were then tilted by $45^{\circ}$ to 148 provide orthogonality of the chemical shift and coupling constant axes and subsequently 149 symmetrised about the F1 axis. ${ }^{1} \mathrm{H}-{ }^{1} \mathrm{H}$ Correlation Spectroscopy (COSY) was performed on 150 all 3 types of pooled biofluid samples in order to detect correlations between protons on 151 adjacent carbons. Transients were acquired with 4096 data points (sweep width of $7200 \mathrm{~Hz}$ in 152 both axis) with 64 scans per increment and 320 increments in the F1 axis. The relaxation 153 between successive pulse cycles was $2.3 \mathrm{~s}$ and were weighted using a sine bell function in $\mathrm{T}_{1}$

154 and $\mathrm{T}_{2}$ prior to fourier transformation and subsequently symmetrised about the diagonal axis.

155 Two-dimensional echo/anti-echo ${ }^{1} \mathrm{H}^{13}{ }^{13} \mathrm{C}$ heteronuclear single quantum correlation (HSQC) 156 spectra were also obtained. 256 scans were collected (16 dummy scans) at a spectral 157 resolution of $4 \mathrm{k}$ in $\mathrm{F} 2$ across a spectral width of $12 \mathrm{ppm}$ for ${ }^{1} \mathrm{H}$ and $170 \mathrm{ppm}$ for the ${ }^{13} \mathrm{C}$ axes.

158 An acquisition time of $0.852 \mathrm{~s}$ and a relaxation delay of $1.2 \mathrm{~s}$ were used and delays were set 159 for a $145 \mathrm{~Hz}$ one bond ${ }^{1} \mathrm{H}-^{13} \mathrm{C}$ coupling constant. Spectra were zero-filled in the $\mathrm{F} 2$ 160 dimension by a factor of 2 to $8 \mathrm{k}$, and zero-filling and linear prediction was applied in F1 to 161 result in a resolution of $1 \mathrm{k}$.

162 NMR spectral data pre-processing

163 Data [-1.0 to $10.0 \mathrm{ppm}$ ] were imported into MatLab environment (7.0 The Mathsworks ${ }^{\mathrm{c}}$ ), 164 where they were automatically phased, baseline-corrected and referenced to TSP $(\delta 0.00)$ or 165 glucose $(\delta$ 5.233) for plasma using scripts written in-house [20]. To reduce analytical 166 variation between samples the residual water signal $(4.67-4.98 \mathrm{ppm})$ was truncated from the 
167 data set. Probabilistic-quotient normalisation was used on each biofluid class separately to

168 account for differing sample dilutions [21].

169 Data analysis of biological matrices

170 Unsupervised multivariate analysis was undertaken to visualise clustering and differences

171 between samples [22]. Principal component analysis (PCA) was constructed using unit-

172 variance scaled data (UV) [23]..

173 Metabolite assignment of endogenous metabolites was made by compiling the following

174 information from each peak: the chemical shift and relative integral height from 1D spectra,

175 the multiplicity using J-res spectra, the proton coupling information from COSY spectra and

176 carbon shifts from HSQC spectra. This information was then used to search for matching

177 metabolites from in-house databases, online databases (http://www.hmdb.ca/) and reference

178 to published literature data [24-26]. Statistical Total Correlation Spectroscopy (STOCSY)

179 was also employed to aid metabolite identification [27]. This method uses statistical

180 connectivity between data points within a spectral profile. However, unlike 2D-NMR this

181 method will also pick up metabolites involved in the same pathways due to biological

182 covariance [28].

183

184 Results

185 Assignment of dominant metabolites across easily obtainable biofluids yields a preliminary

186 equine metabolic atlas

187 The majority of known NMR-detectable metabolites were assigned in each biological matrix

188 (Fig 1), with numerical reference to Table 1. Metabolite assignments were performed using 189 two-dimensional correlation (COSY) and J-resolved (J-res) spectroscopy to ascertain peak 
190 multiplicity, coupling constants and to overcome peak overlap. This is demonstrated in the

191 COSY (Fig 2A) and the J-res (Fig 2B). Heteronuclear Single Quantum Coherence (HSQC)

192 was also performed to provide ${ }^{13} \mathrm{C}$ shifts and confirm assignments. Putative metabolite

193 assignment was made using ${ }^{1} \mathrm{H}$ and ${ }^{13} \mathrm{C}$ chemical shifts, peak multiplicity, coupling constants

194 and relative peak integrals. Overall, 102 metabolites were identified by ${ }^{1} \mathrm{H}-\mathrm{NMR}$ in the 3

195 biofluids (Table 1). Detailed assignment information is shown in Supplementary Item 2.

196

197 Cross-compartmental analysis revealed a core metabonome, along with compartment specific

198 metabolites

199 Metabolites assigned to the 3 biofluids were compared to ascertain ubiquitous metabolites

200 and those that were specific to each biological compartment. The metabolic variation across

201 the biological matrices is displayed in a Venn diagram (Fig 3) to easily visualise inter-

202 compartmental overlap. A total of 14 metabolites were ubiquitous to all biofluids, which we

203 will refer to as 'core' metabolites. These include energy-related metabolites such as glucose

204 and lactate as well as a number of amino-acids including alanine, arginine, glycine,

205 glutamine, taurine, threonine and valine. The microbial related metabolites acetate, formate

206 and $p$-hydroxyphenylacetate were also conserved across all biofluids studied.

207 PCA revealed that inter-animal metabolic variation was lower than the variation between the

208 different biofluids as visualised via clustering in the PCA scores plot (Fig 4A). As would be

209 expected, the PCA samples cluster based on biofluid type. However, importantly the degree

210 of clustering is different amongst biological matrices. Faecal samples demonstrate that they

211 are inherently variable compared to either matched urine or plasma samples. The faecal

212 metabonome displayed relatively higher concentrations of SCFA (butyrate, acetate and

213 propionate) compared to plasma and urine. Urine samples had relatively higher level of gut- 
214 microbial co-metabolites such as hippurate, phenylacetylglycine (PAG), p-cresyl sulfate and

215 trimethylamine- $N$-oxide (TMAO). In contrast, energy-related metabolites such as glucose and 216 pyruvate were observed in relatively higher concentrations in plasma compared to urine.

217 Variance plots shown in Fig 4A and B display the mean spectra of faeces and urine 218 respectively coloured by the variance, represented as a percentage of the total variance. Here, 219 creatinine can be seen to represent over $10 \%$ of the total variance seen within the urinary 220 profiles (Fig 4B) and acetate accounted for over $28 \%$ of the total variance observed in the

221 faecal profile (Fig 4A) compared to $1.8 \%$ in the plasma profile (not shown).

222

223 Plasma

224 Equine plasma contained relatively few metabolites compared to urine and faeces when 225 analysed by NMR spectroscopy, partly as a result of the overlap of signals from 226 macromolecular components such as lipoproteins and low molecular weight chemicals. A 227 total of 38 metabolites were identified (Table 1), and of these 12 were unique to this biofluid, 228 including the amino-acids tyrosine and phenylalanine and ketone bodies ( $\alpha$ - and $\beta$ 229 hydroxybutyrate). Plasma was observed to contain the highest levels of glucose compared to 230 other biofluids within the data matrix.

231 Urine

232 The equine urinary metabolic profile was the most metabolically abundant of all biofluids 233 measured (Table 1). In total 65 metabolites were identified. Of these, 11 were unique to urine 234 and plasma, 3 were present in both urine and faeces and 39 metabolites were specific to 235 urine. Urinary-specific metabolites included a number of aromatic compounds that arise from 236 microbial-host co-metabolism. Hippurate, PAG, p-cresyl glucuronide and sulphate were 
237 notably prominent in the aromatic region of the horse urine spectra (Fig 1B). Other urine

238 specific metabolites of note include dietary compounds such as proline betaine.

239 Faeces

240 The faecal metabotype contained 43 metabolites, including 21 specific to faeces (Fig 3).

241 These metabolites include microbial fermentation products, SCFA (butyrate and propionate),

242 and a number of dietary metabolites (caprylate and arabinose, maltose, glycerol and xylose).

243 Acetate was present in significantly greater concentrations than other metabolites.

244

245 Discussion

246 This is the first systematic description of the dominant metabolites of the healthy

247 Thoroughbred racehorse and is important in establishing a metabolic reference from which to

248 compare pathology-related variation. Characterisation of the equine metabolome highlights

249 the diversity of information available in different biological matrices and as such provides a

250 useful guide for researchers. Importantly, as has been shown in other species, urine provides

251 a biological window into host-microbial metabolic interactions in the horse [8]. Although

252 faeces may be considered a more direct representation of microbial metabolism, Fig 4A and

253 B highlights the relatively greater variation in the faecal metabolic profiles compared to

254 plasma and urine. Additionally, microbial-derived compounds absorbed from the gut are

255 commonly not well represented in the faecal signature rendering the biological usefulness of

256 this biofluid in gastrointestinal disease and mammalian-microbial co-metabolsim

257 questionable.

258 A ubiquitous metabonome is present amongst mammals. The core equine metabonome

259 comprises metabolites present in all 3 biofluids studied. 14 metabolites were identified

260 including amino-acids, gut microbial metabolites and energy metabolites. Many of these 
261 metabolites are ubiquitous, with varied functions and located in numerous tissue types.

262 Similar findings were reported by Merrifield et al. with 22 metabolites shared across 4 263 biological matrices in pigs [8].

264 Marked ${ }^{1} \mathrm{H}-\mathrm{NMR}$ metabolic similarities between horses and humans are suggested by 265 observations of a number of shared metabolites. In total, there were 32 plasma, 23 urine and 26627 faecal NMR detectable compounds common to both horses and humans [29-31].

267 Metabolic consistency was strongest across the plasma profiles. This validates the concept of

268 conservation of mammalian physiology across species and that homeostatic metabolic control

269 is tight in both systems. A lower percentage of the equine faecal and urine metabolic profiles

270 were shared with humans but this was to be expected due to different digestive systems and

271 metabolic pathways. In contrast, ruminants share a number of gut microbial co-metabolites

272 such as hippurate and PAG [32-34]. These herbivores have similar digestive strategies to

273 obtain nutrients from a cellulose-rich diet.

274 Excretory biofluids (urine and faeces) are the most metabolite-rich. The environmentally-

275 determined nature of these biofluids renders them under less tight homeostatic control than

276 that of the plasma. However, urine was also remarkably tightly controlled, given the

277 relatively homogeneous genetic and environmental backgrounds of Thoroughbred racehorses.

278 Differing creatinine excretion in one animal (due to the sample being collected after exercise)

279 was responsible for the increased inter-animal variation observed (Fig 4A). In urine, 39

280 metabolites were identified as being unique whereas 21 metabolites were faeces-specific and 281 metabolites were plasma-specific. However, there were a small number of low282 concentration unassigned metabolites that have not been included in these counts. All 3 283 biofluids are likely to contain thousands of metabolites which are too dilute to produce a 284 significant NMR signal or were not detected by NMR in this study, but we feel we were able 
to capture a good representation of the normal equine metabonome and this has been verified

286 by our ongoing work on a larger cohort of animals (data not shown).

287 Inter-compartmental variation is greater than inter-animal variation and is consistent with

288 other mammalian studies [8; 35]. Faecal samples varied from other biological matrices due to

289 the presence of a number of SCFAs, formate and isovalerate. SCFAs are the product of gut-

290 microbial fermentation of dietary fibre and contribute up to $70 \%$ of a horse's energy

291 requirements [36;37]. Acetate level variability is likely to be due to differing bacterial

292 communities and consequently SCFA production. Collection time in relation to feeding time

293 is known to exert an effect on SCFA levels in equine faeces [38]. Samples were consistently

294 taken before a morning feed. However, this variability could be due to the difference in

295 individual intestinal transit times [39; 40]. Other SCFAs were not seen to vary to the same

296 extent as acetate. Butyrate is the main energy source for colonocytes and hence is likely to

297 have been utilised rather than excreted [41]. Butyrate and propionate are extensively

298 metabolised by first-pass metabolism and therefore absent in NMR detectable quantities in

299 urine and plasma samples. Creatinine was the greatest source of variation amongst urine

300 samples. Creatinine is a waste product of muscle metabolism formed from creatine in order to

301 maintain ATP levels during exercise [42]. The concentration of urine and thus metabolites

302 can change dramatically. Normalisation of the data prior to analysis helps to minimise

303 spectral anomalies caused by differences in urinary dilution. Creatinine levels can vary

304 according to factors such as muscle mass, physical exercise, diet, age and muscle damage

305 from previous strenuous exercise [43].

306 The urine metabolome provides a metabolic window into gut microbial co-metabolism.

307 Urine was found to differ from the other biological matrices due to the presence of a number 308 of gut-microbial co-metabolites (hippurate, PAG and $p$-cresyl sulphate and TMAO). These 
309 compounds originate from exogenous sources (microbial and dietary) and are incorporated

310 into the host circulation after absorption. They subsequently undergo enzymatic conjugation

311 in the liver and gut mucosa to increase their polarity and enable renal excretion. In mammals,

312 enterohepatic recycling means further metabolism can occur at the gut-level [44]. Hippurate

313 is an aromatic compound predominantly formed from glycine conjugation of dietary or

314 microbial benzoate and PAG is a glycine-conjugated microbial metabolite of phenylalanine

315 metabolism (glutamine in higher apes and humans) [45]. $P$-cresol is formed from bacterial

316 degradation of tyrosine and is subsequently sulfated or glucuronidated in the liver or gut [46].

317 Both forms of conversion occur in rodents and horses, whereas humans predominately form

318 sulphate conjugates and pigs predominately glucuronidate the cresol molecule.

319 Trimethylamine (TMA) is a microbial degradation product of dietary choline; this metabolite

320 is absorbed from the gut and subsequently oxidised in the liver to produce TMAO [47]. A

321 number of anaerobic bacterial populations are known to produce these metabolites including

322 clostridia [48-50]. This taxonomic classification of bacteria has been associated with

323 intestinal disease in horses as well as being part of the normal microflora [51-54].

$324{ }^{1} \mathrm{H}$ NMR spectroscopy is a robust method for assessing the inter-animal variation in

325 Thoroughbred racehorses. It is important to address potential sources of variation when

326 assessing metabonomic studies. The sensitive nature of such investigations necessitates the

327 collection of metadata to help explain possible variation between the samples. This

328 information should include details from sample collection, sample storage and run order, as

329 well as information relating to sample subject (health status, age, sex for example). The effect

330 of these is widely reported in the literature $[35 ; 55 ; 56]$. Although the 7 horses were taken

331 from 2 different yards, the samples clustered tightly, highlighting the metabolic uniformity of 332 these Thoroughbred racehorses despite differing age ranges, location and stages of fitness 
333 (see Supplementary Item 1). This study was intended to create a reference tool for research

334 into Thoroughbred racehorse metabolism and microbial co-metabolism. Since the samples 335 from this first pilot clustered closely for all 3 biofluids indicating that the dominant 336 metabolites visible by NMR were conserved across animals, it was deemed unnecessary to 337 collect samples from further animals to minimise unnecessary sampling. Although, the 338 sample size in this study is small and only one metabolic snap shot was taken for each horse, 339 the fact that inter-animal variation in metabolic profiles was low, suggests that 'healthy'

340 Thoroughbreds share a similar metabolic phenotype, which we explored using a range of 341 NMR-based structural elucidation tools including 2-D pulse sequences to elicit carbon-proton

342 correlations and statistical spectroscopy methods. Our future work includes a larger study

343 investigating normal variation amongst different racehorse populations over time.

344 This work comprehensively assigns dominant features of the ${ }^{1} \mathrm{H}$ NMR spectra of the equine 345 metabonome from plasma, urine and faeces and for the first time provides baseline 346 information for future studies in equine health and disease. Urine and faecal profiles provide 347 an insight into host-microbial metabolic interactions, whereas plasma profiles are more likely 348 to represent host physiological processes. The purpose of this study is to provide an analytical 349 template to researchers thinking about adding metabonomic analysis to their experiments and 350 to indicate which biofluids may be of use to them. We have showed that faecal samples are 351 more variable that either urine or plasma. Plasma is under tight homeostatic control and thus 352 might be expected to show relatively less variation. However, urine, other than one identified 353 outlier, exhibited less variation than the other biofluids under investigation and contributed 354 the greatest number of identifiable metabolites. Further studies are warranted using 355 metabonomic and metagenomic technology to explore the role of gut microbes on equine 356 physiology and metabolism. 
359 Authors' declaration of interests

360 No competing interests have been declared.

361

362 Source of funding

363 Horserace Betting Levy Board Research Scholarship at Imperial College London.

364

365 Ethical Animal Research

366 Sampling and metadata collection were carried out under University of Liverpool ethics

367 approval RETH000363, with the informed consent of the trainer.

368

369 Acknowledgements

370 We are grateful to the racehorse trainers and owners who allowed us to sample their horses,

371 to Elizabeth Metcalfe from the Animal Health Trust, Newmarket for technical assistance and

372 Aisling Glennie at Boehringer Ingelheim for help editing the manuscript.

373

374

375 Authorship

376 E. Escalona contributed to all sections. J. Leng contributed to study execution, and data

377 analysis and interpretation. J. Swann and A. Dona contributed to study design, study

378 execution, and data analysis and interpretation. C. Merrifield contributed to the preparation of 379 the manuscript. All authors gave their final approval of the manuscript.

380

381 


\section{Figure legends:}

385 Fig 1: (A) $700 \mathrm{MHz} 1 \mathrm{D}{ }^{1} \mathrm{H}-\mathrm{NMR}$ spectrum of urine, (B) $\mathrm{CPMG}{ }^{1} \mathrm{H}-\mathrm{NMR}$ spectrum of 386 plasma, (C)1D ${ }^{1} \mathrm{H}-\mathrm{NMR}$ spectrum of faecal water. All spectra partially labelled according to 387 the assignments made in Table 1 and Supplementary Item 2.

388

389 Fig 2: (A) $800 \mathrm{MHz}{ }^{1} \mathrm{H}$ COSY NMR spectrum of the aromatic region of urine (B) J-res NMR

390 spectrum of plasma highlighting the aliphatic region between 3-4.5 ppm. Key to metabolite

391 identification is provided in Table 1 and Supplementary Item 2.

392

393 Fig 3: (A) Venn diagram highlighting the degree of metabolite overlap between biological 394 compartments. The central section represents the number of core metabolites visible across 395 all biological matrices (14 ubiquitous metabolites). The outer circle with numbered slices 396 represents biological matrix specific metabolites that are ordered according to

397 origins/function, and numbered according to metabolites in Table 1. Compartments are 398 colour coded - plasma (red), urine (yellow) and faeces (green). Coloured dots represent 399 metabolic functions, protein and amino-acid metabolism (purple), energy metabolism 400 (orange) and fat metabolism (blue). Asterisk denotes metabolite can be mammalian in origin 401 and a ' $d$ ' denotes metabolite can be from dietary origin.

402

403 Fig 4: (A) PCA scores plot demonstrating increased faecal variability relative to other 404 biological matrices. Plasma (red), urine (yellow) and faecal (green) samples. Principal 405 component 1 (PC1) accounts for 51\%, PC2 for 15\%, and PC3 for 5\% of the total variation. 406 Variance colour plots indicating the percentage of the total variance for each metabolite in 407 (B) faeces and (C) urine (Mean spectrum is plotted, coloured by variance expressed as a 408 percentage of the total variance).

409 
410 Table 1: Metabolites identified using ${ }^{1} \mathrm{H}-\mathrm{NMR}$ of equine plasma, urine and faeces. 411 Metabolite numbers correspond with annotated ${ }^{1} \mathrm{H}-\mathrm{NMR}$ spectra in Figs 1, 2 and 3. 412 Metabolites are assigned to biofluids denoted by coloured dots; urine (yellow), plasma (red), 413 faeces (green). The 14 core metabolites are highlighted in purple.

414

415

416 Manufacturers' addresses

$417{ }^{a}$ Bertin Technologies, Montigny-le-Bretonneux, France

$418{ }^{\mathrm{b}}$ Bruker, Massachusetts, USA

$419{ }^{\mathrm{c} M A T L A B}$, Mathwork, Massachusetts, USA

420

421 Additional Supplementary Items may be found in the online version of this article at the 422 publisher's website:

423

424 Supplementary Item 1: The table highlights sample metadata and possible sources of 425 variation within the data set. Yard, gender, age and training schedule are included. Gender is 426 denoted as $\mathrm{G}=$ gelding, $\mathrm{C}=$ colt.

427

428 Supplementary Item 2: Table showing metabolites found in plasma (red circle), urine

429 (yellow circle) and faeces (green circle) using ${ }^{1} \mathrm{H}-\mathrm{NMR}$ spectroscopy. Peak multiplicities and 430 chemical shifts are shown and structural information is also provided. $\uparrow$ indicates tentative 431 assignment. Details on each metabolite's origin and function are highlighted as well as a link 432 to the metabolite's page in the hmdb database.

433

434 Supplementary Item 3: Table denoting feeding regimes on the 2 yards sampled. Top section 435 highlights concentrate feeds and bottom highlights roughage types offered.

436

437 Supplementary Item 4: $800 \mathrm{MHz}{ }^{1} \mathrm{H}$ COSY NMR spectrum of the aliphatic region of 438 faeces, between 3-4 ppm. Key to metabolite identification is provided in Table 1 and 439 Supplementary Item 2.

440 
Supplementary Item 5: $800 \mathrm{MHz}{ }^{1} \mathrm{H}^{13} \mathrm{C}$ HSCQ NMR spectrum of the aromatic region of

443 Supplementary Item 2.

\section{References}

445

[1] Nicholson, J.K., Lindon, J.C. and Holmes, E. (1999) 'Metabonomics': understanding

446 the metabolic responses of living systems to pathophysiological stimuli via

447 multivariate statistical analysis of biological NMR spectroscopic data. Xenobiotica 29, 1181-1189.

451

[2] Zhang, S., Nagana Gowda, G.A., Asiago, V., Shanaiah, N., Barbas, C. and Raftery, D.

[4] Gowda, G.A., Zhang, S., Gu, H., Asiago, V., Shanaiah, N. and Raftery, D. (2008) (2008) Correlative and quantitative 1H NMR-based metabolomics reveals specific metabolic pathway disturbances in diabetic rats. Anal. Biochem. 383, 76-84.

[3] Poli, D., Carbognani, P., Corradi, M., Goldoni, M., Acampa, O., Balbi, B., Bianchi, L., Rusca, M. and Mutti, A. (2005) Exhaled volatile organic compounds in patients with non-small cell lung cancer: cross sectional and nested short-term follow-up study. Respir. Res. 6, 71.

466

467

468

469

470

471

472

473

474

475 Metabolomics-based methods for early disease diagnostics. Expert Rev. Mol. Diagn.

$\mathbf{8}, 617-633$.

[5] Peng, J.-B., Jia, H.-M., Xu, T., Liu, Y.-T., Zhang, H.-W., Yu, L.-L., Cai, D.-Y. and Zou, Z.-M. (2011) A (1)H NMR based metabonomics approach to progression of coronary atherosclerosis in a rabbit model. Process Biochemistry 46, 2240-2247.

\section{[7] Saric, J., Wang, Y., Li, J., Coen, M., Utzinger, J., Marchesi, J.R., Keiser, J., Veselkov,} K., Lindon, J.C., Nicholson, J.K. and Holmes, E. (2008) Species variation in the fecal metabolome gives insight into differential gastrointestinal function. Journal of Proteome Research 7, 352-360.

[8] Merrifield, C.A., Lewis, M., Claus, S.P., Beckonert, O.P., Dumas, M.-E., Duncker, S., Kochhar, S., Rezzi, S., Lindon, J.C., Bailey, M., Holmes, E. and Nicholson, J.K. (2011) A metabolic system-wide characterisation of the pig: a model for human physiology. Molecular Biosystems 7, 2577-2588.

[9] He, Q., Ren, P., Kong, X., Wu, Y., Wu, G., Li, P., Hao, F., Tang, H., Blachier, F. and Yin, Y. (2012) Comparison of serum metabolite compositions between obese and lean Yrowing pigs using an NMR-based metabonomic approach. Journal of Nutritional Biochemistry 23, 133-139. 
Scarth, J.P., Spencer, H.A., Timbers, S.E., Hudson, S.C. and Hillyer, L.L. (2010) The use of in vitro technologies coupled with high resolution accurate mass LC-MS for studying drug metabolism in equine drug surveillance. Drug Testing and Analysis 2, $1-10$.

[11] Liu, Y., Uboh, C.E., Soma, L.R., Li, X., Guan, F., You, Y. and Chen, J.-W. (2011) Efficient Use of Retention Time for the Analysis of 302 Drugs in Equine Plasma by Liquid Chromatography-MS/MS with Scheduled Multiple Reaction Monitoring and Instant Library Searching for Doping Control. Analytical Chemistry 83, 6834-6841.

[12] Hodavance, M.S., Ralston, S.L. and Pelczer, I. (2007) Beyond blood sugar: the potential of NMR-based metabonomics for type 2 human diabetes, and the horse as a possible model. Analytical and Bioanalytical Chemistry 387, 533-537.

[13] Keller, M.D., Pollitt, C.C. and Marx, U.C. (2011) Nuclear magnetic resonance-based metabonomic study of early time point laminitis in an oligofructose-overload model. Equine Vet. J. 43, 737-743.

[14] Ralston, S.L., Pappalardo, L. and Pelczer, I. (2011) Breed and age effects on metabolic profiles of young horses using NMR-based Metabonomic analyses of serum. Journal of Equine Veterinary Science 31, 304-305.

\section{[15] Ralston, S.L., Pelczer, I., Shatynski, C. and Spears, P.F. (2009) NMR-based} Metabonomic Analysis of Serum From Standardbred Yearlings With or Without Hock Osteochondrosis Dessicans Lesions: Preliminary Study. Journal of Equine Veterinary Science 29, 385-386.

[16] Pappalardo, L., Pelczer, I. and Ralston, S.L. (2013) Metabolic Differences between Draft-cross and Mustang Horses Detected by Metabonomic Analyses. Journal of Equine Veterinary Science 33, 1044-1049.

\section{[17] Dumas, M.E., Maibaum, E.C., Teague, C., Ueshima, H., Zhou, B.F., Lindon, J.C.,} Nicholson, J.K., Stamler, J., Elliott, P., Chan, Q. and Holmes, E. (2006) Assessment of analytical reproducibility of H-1 NMR spectroscopy based metabonomics for large-scale epidemiological research: the INTERMAP study. Analytical Chemistry 78, 2199-2208.

[18] Beckonert, O., Keun, H.C., Ebbels, T.M.D., Bundy, J.G., Holmes, E., Lindon, J.C. and Nicholson, J.K. (2007) Metabolic profiling, metabolomic and metabonomic procedures for NMR spectroscopy of urine, plasma, serum and tissue extracts. Nature Protocols 2, 2692-2703.

[19] Wright, B., Greatbanks, D., Taberner, J. and Wilson, I.D. (1995) Comparison of Three Methods for Water Suppression in Biofluid NMR: Advantages of NOESYPRESAT. Pharmacy and Pharmacology Communications 1, 197-199. 
531 [20] Pearce, J.T.M., Athersuch, T.J., Ebbels, T.M.D., Lindon, J.C., Nicholson, J.K. and Keun, H.C. (2008) Robust algorithms for automated chemical shift calibration of 1D H-1 NMR spectra of blood serum. Analytical Chemistry 80, 7158-7162.

[21] Dieterle, F., Ross, A., Schlotterbeck, G. and Senn, H. (2006) Probabilistic quotient normalization as robust method to account for dilution of complex biological mixtures. Application in H-1 NMR metabonomics. Analytical Chemistry 78, 42814290. Journal of Proteome Research 6, 469-479.

[23] Craig, A., Cloareo, O., Holmes, E., Nicholson, J.K. and Lindon, J.C. (2006) Scaling and normalization effects in NMR spectroscopic metabonomic data sets. Analytical

[24] Fan, T.W.M. (1996) Metabolite profiling by one- and two-dimensional NMR analysis

[26] Bollard, M.E., Keun, H.C., Beckonert, O., Ebbels, T.M.D., Antti, H., Nicholls, A.W., of complex mixtures. Progress in Nuclear Magnetic Resonance Spectroscopy 28, 161-219.

[25] Nicholson, J.K. and Wilson, I.D. (1989) High resolution proton magnetic resonance spectroscopy of biological fluids. Progress in Nuclear Magnetic Resonance Spectroscopy 21, 449-501.

560 [27] Cloarec, O., Dumas, M.E., Craig, A., Barton, R.H., Trygg, J., Hudson, J., Blancher, 561 C., Gauguier, D., Lindon, J.C., Holmes, E. and Nicholson, J. (2005) Statistical total correlation spectroscopy: An exploratory approach for latent biomarker identification from metabolic H-1 NMR data sets. Analytical Chemistry 77, 1282-1289. Spectroscopic correlation analysis of NMR-based metabonomics in exercise science. Analytica Chimica Acta 652, 173-179.

[29] Nicholson, J.K., Foxall, P.J., Spraul, M., Farrant, R.D. and Lindon, J.C. (1995) 750 MHz 1H and 1H-13C NMR spectroscopy of human blood plasma. Anal. Chem. 67, 793-811.

[30] Holmes, E., Foxall, P.J., Spraul, M., Farrant, R.D., Nicholson, J.K. and Lindon, J.C. (1997) $750 \mathrm{MHz}$ 1H NMR spectroscopy characterisation of the complex metabolic pattern of urine from patients with inborn errors of metabolism: 2-hydroxyglutaric aciduria and maple syrup urine disease. J. Pharm. Biomed. Anal. 15, 1647-1659. 
[31] Le Gall, G., Noor, S.O., Ridgway, K., Scovell, L., Jamieson, C., Johnson, I.T., Colquhoun, I.J., Kemsley, E.K. and Narbad, A. (2011) Metabolomics of Fecal Extracts Detects Altered Metabolic Activity of Gut Microbiota in Ulcerative Colitis and Irritable Bowel Syndrome. Journal of Proteome Research 10, 4208-4218. Ametaj, B.N., Zebeli, Q., Saleem, F., Psychogios, N., Lewis, M.J., Dunn, S.M., Xia, J.G. and Wishart, D.S. (2010) Metabolomics reveals unhealthy alterations in rumen metabolism with increased proportion of cereal grain in the diet of dairy cows. Metabolomics 6, 583-594.

[33] Nyberg, N.T., Nielsen, M.O. and Jaroszewski, J.W. (2010) Metabolic trajectories based on H-1 NMR spectra of urines from sheep exposed to nutritional challenges

[34] Li, J., Wijffels, G., Yu, Y., Nielsen, L.K., Niemeyer, D.O., Fisher, A.D., Ferguson,

[37] Cummings, J.H. and Macfarlane, G.T. (1997) Role of intestinal bacteria in nutrient D.M. and Schirra, H.J. (2011) Altered fatty acid metabolism in long duration road transport: An NMR-based metabonomics study in sheep. J. Proteome Res. 10, 1073 1087.

[35] Bollard, M.E., Stanley, E.G., Lindon, J.C., Nicholson, J.K. and Holmes, E. (2005) NMR-based metabonomic approaches for evaluating physiological influences on biofluid composition. Nmr in Biomedicine 18, 143-162.

606

607 [38] Desrousseaux, G., Santos, A.S., Pellikaan, W.F., Van der Poel, A.F.B., Cone, J.W.,

612 [39] Van Weyenberg, S., Sales, J. and Janssens, G.P.J. (2006) Passage rate of digesta

613

614

615 [40] Hudson, N.P.H. and Merritt, A.M. (2008) Equine gastrointestinal motility research:

616

617

618 [41] Bugaut, M. and Bentejac, M. (1993) Biological effects of short-chain fatty acids in

621 [42] Wyss, M. and Kaddurah-Daouk, R. (2000) Creatine and creatinine metabolism. Guedes, C.M.V., Ferreira, L.M.M. and Rodrigues, M.A.M. (2012) Effect of collection time on the fermentative activity of microbes in equine faeces. Animal Feed Science and Technology 178, 183-189. 
[43] Baxmann, A.C., Ahmed, M.S., Marques, N.C., Menon, V.B., Pereira, A.B., Kirsztajn, G.M. and Heilberg, I.P. (2008) Influence of muscle mass and physical activity on serum and urinary creatinine and serum cystatin C. Clinical Journal of the American Society of Nephrology 3, 348-354.

630

631

632

$633 \sim[45]$

634

[44] Nicholson, J.K., Holmes, E. and Wilson, I.D. (2005) Gut microorganisms, mammalian metabolism and personalized health care. Nature Reviews Microbiology 3, 431-438.

635 Lees, H.J., Swann, J.R., Wilson, I.D., Nicholson, J.K. and Holmes, E. (2013)

636

637

638

639

640

641

642

643

644

645

646

647

648

649

650

651

652

653

654

655

656

657

658

659

660

661

662

663

664

665

666

667

668

669

670 Hippurate: The Natural History of a Mammalian-Microbial Cometabolite. $J$. Proteome Res. 5, 1527-1546.

[46] Clayton, T.A., Baker, D., Lindon, J.C., Everett, J.R. and Nicholson, J.K. (2009) Pharmacometabonomic identification of a significant host-microbiome metabolic interaction affecting human drug metabolism. Proc. Natl. Acad. Sci. USA 106, 1472814733.

[47] Bennett, B.J., de Aguiar Vallim, T.Q., Wang, Z., Shih, D.M., Meng, Y., Gregory, J., Allayee, H., Lee, R., Graham, M., Crooke, R., Edwards, P.A., Hazen, S.L. and Lusis, A.J. (2013) Trimethylamine-N-oxide, a metabolite associated with atherosclerosis, exhibits complex genetic and dietary regulation. Cell Metab. 17, 49-60.

[48] Waldram, A., Holmes, E., Wang, Y., Rantalainen, M., Wilson, I.D., Tuohy, K.M., McCartney, A.L., Gibson, G.R. and Nicholson, J.K. (2009) Top-Down Systems Biology Modeling of Host Metabotype-Microbiome Associations in Obese Rodents. Journal of Proteome Research 8, 2361-2375.

[49] Peppercorn, M.A. and Goldman, P. (1971) Caffeic acid metabolism by bacteria of the human gastrointestinal tract. J. Bacteriol. 108, 996-1000.

[50] Li, J.V., Ashrafian, H., Bueter, M., Kinross, J., Sands, C., le Roux, C.W., Bloom, S.R., Darzi, A., Athanasiou, T., Marchesi, J.R., Nicholson, J.K. and Holmes, E. (2011) Metabolic surgery profoundly influences gut microbial-host metabolic crosstalk. Gut 60, 1214-1223.

[51] Diab, S.S., Rodriguez-Bertos, A. and Uzal, F.A. (2013) Pathology and diagnostic criteria of Clostridium difficile enteric infection in horses. Vet. Pathol. 50, 1028-1036.

[52] Diab, S.S., Songer, G. and Uzal, F.A. (2013) Clostridium difficile infection in horses: A review. Veterinary Microbiology 167, 42-49.

[53] Costa, M.C., Arroyo, L.G., Allen-Vercoe, E., Stampfli, H.R., Kim, P.T., Sturgeon, A. and Weese, J.S. (2012) Comparison of the fecal microbiota of healthy horses and horses with colitis by high throughput sequencing of the V3-V5 region of the $16 \mathrm{~S}$ rRNA gene. PLoS One 7, e41484. 
[54] MM, O.D., Harris, H.M., Jeffery, I.B., Claesson, M.J., Younge, B., PW, O.T. and Ross, R.P. (2013) The core faecal bacterial microbiome of Irish Thoroughbred racehorses. Lett. Appl. Microbiol. 57, 492-501. Bollard, M.E., Holmes, E., Lindon, J.C., Mitchell, S.C., Branstetter, D., Zhang, W. and Nicholson, J.K. (2001) Investigations into biochemical changes due to diurnal variation and estrus cycle in female rats using high-resolution H-1 NMR spectroscopy 679 of urine and pattern recognition. Analytical Biochemistry 295, 194-202.

681 Saude, E.J. and Sykes, B.D. (2007) Urine stability for metabolomic studies: effects of

682 preparation and storage. Metabolomics 3, 19-27.

683

684

685 


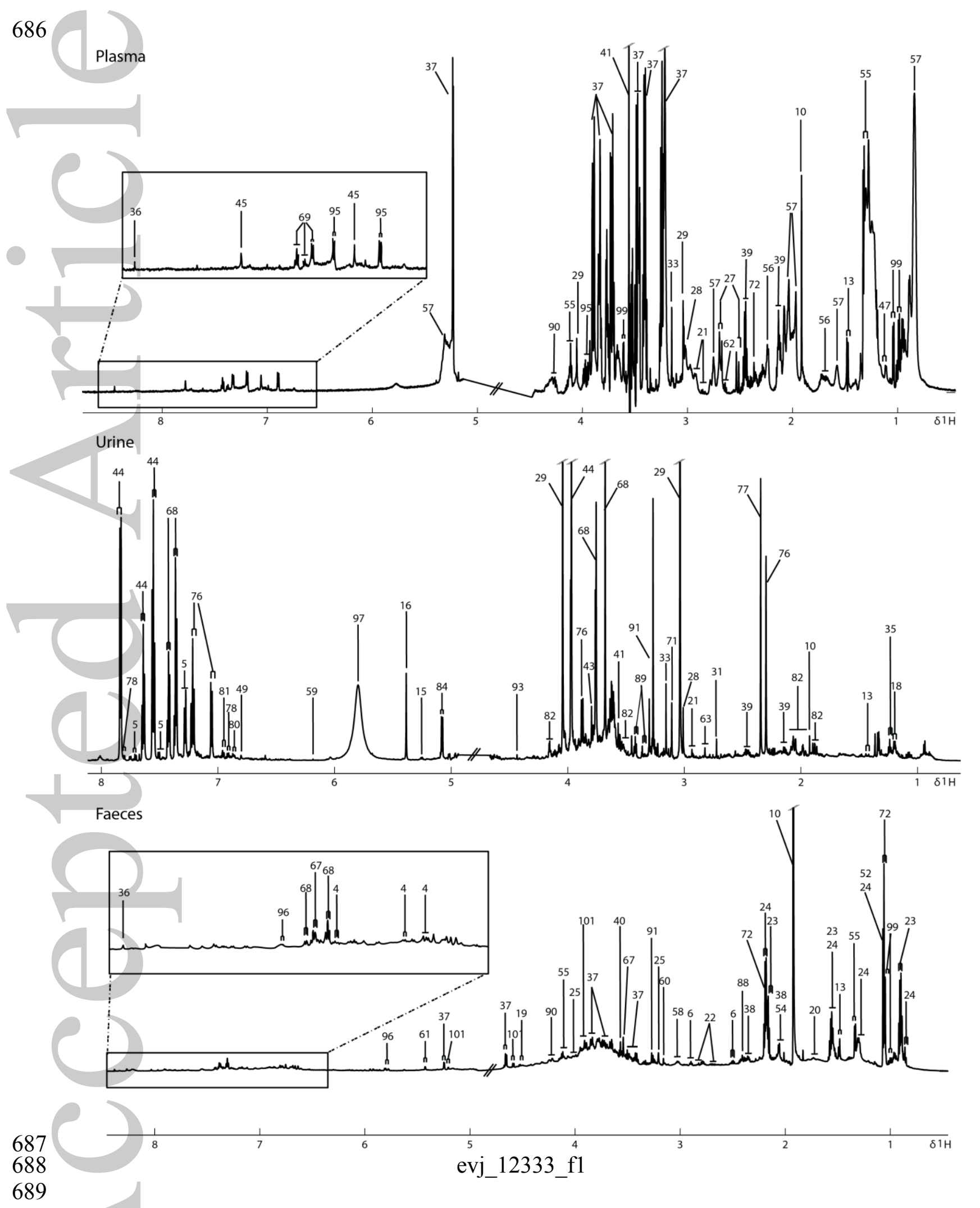




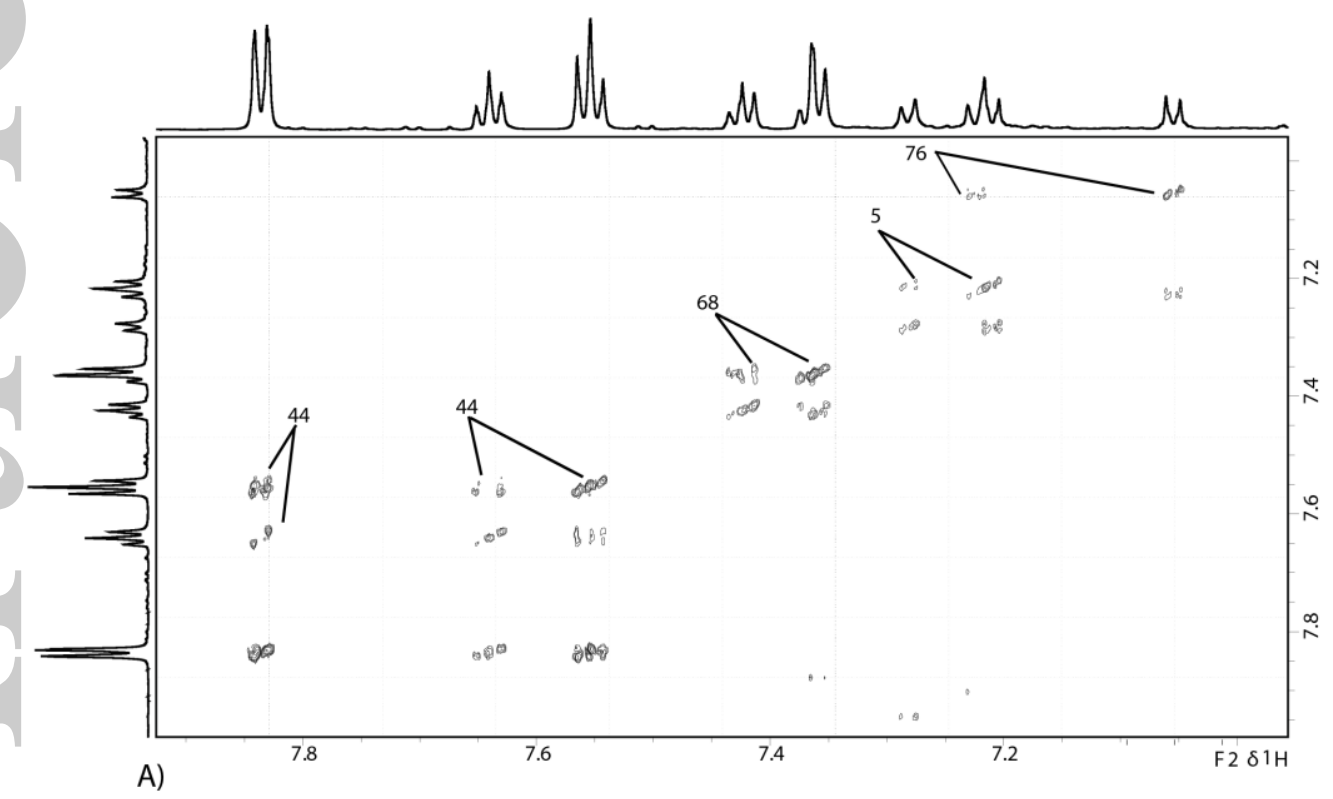

A)

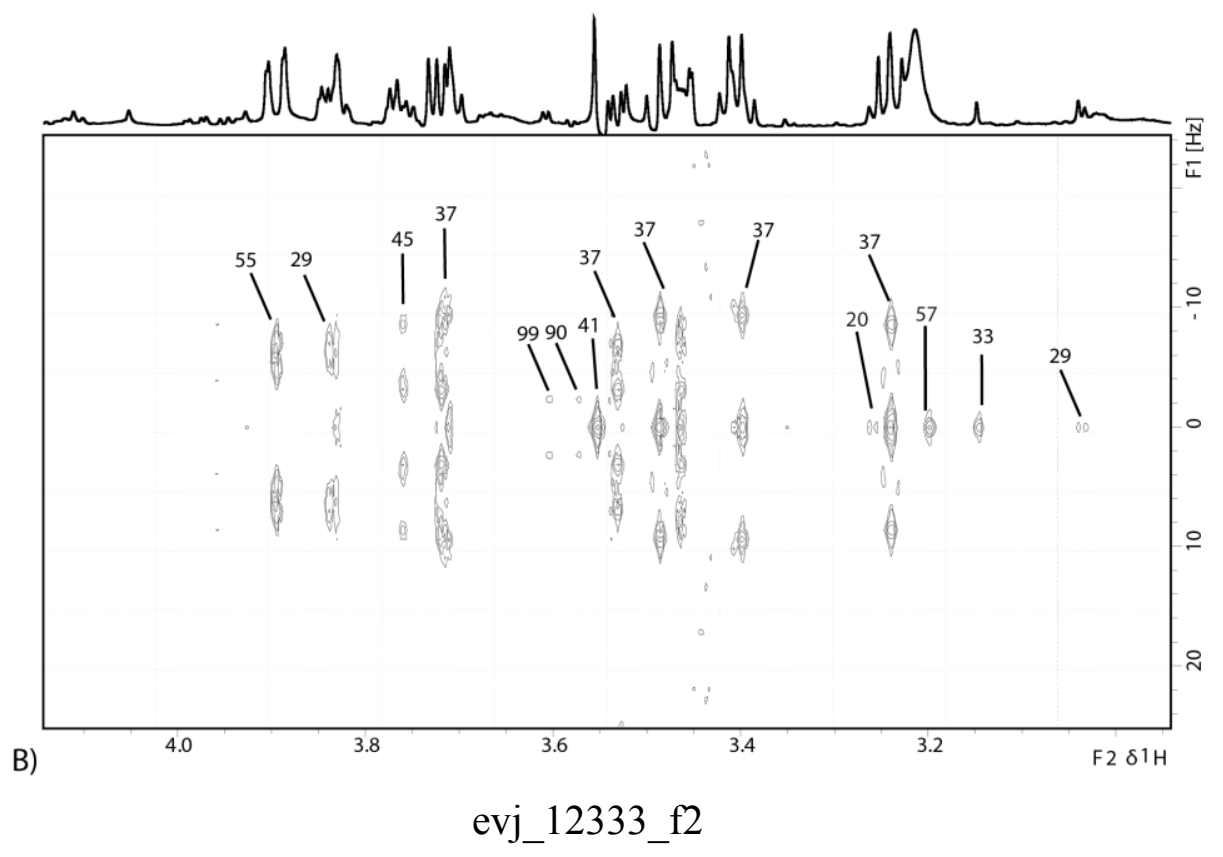

This article is protected by copyright. All rights reserved. 


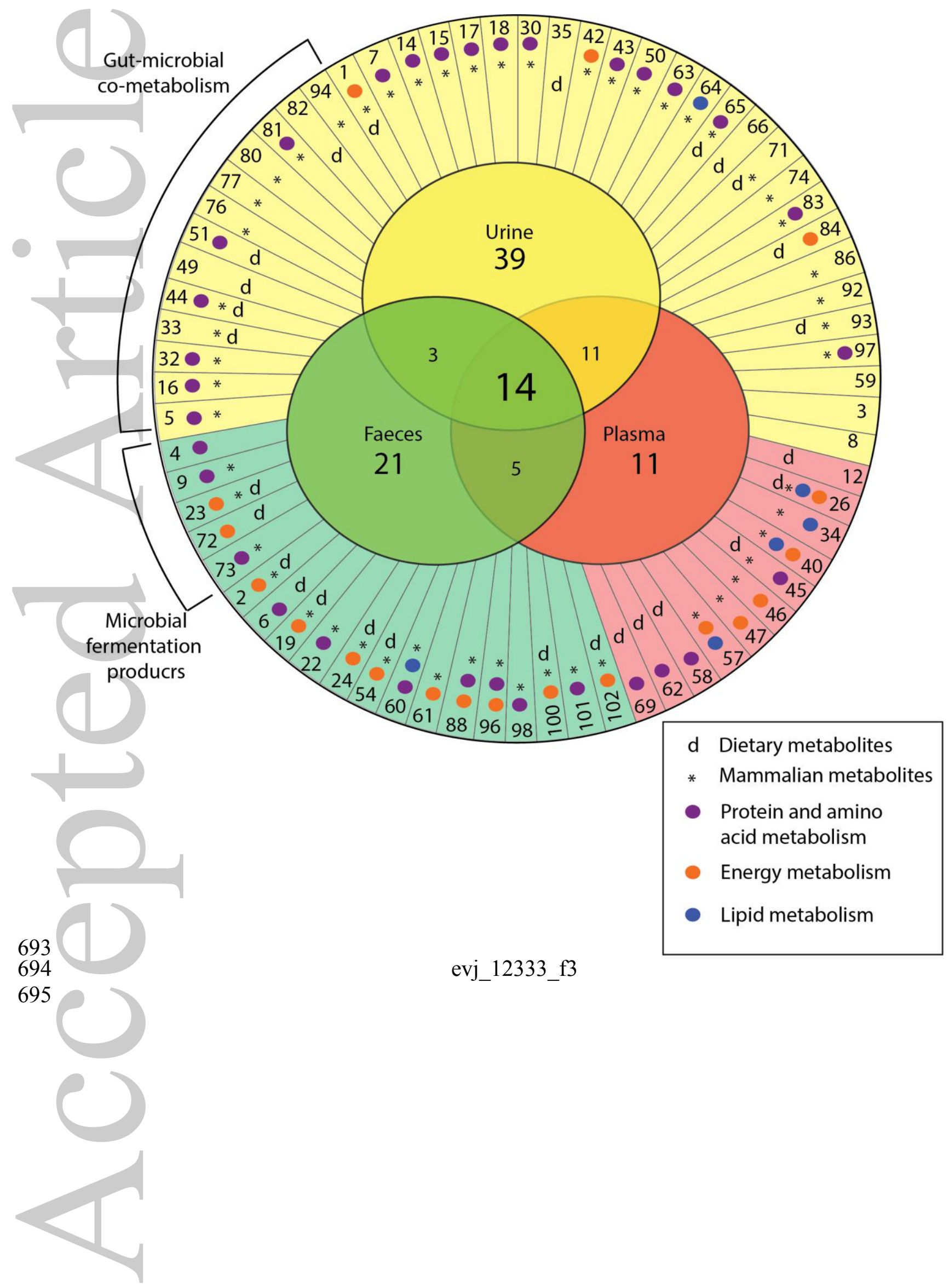



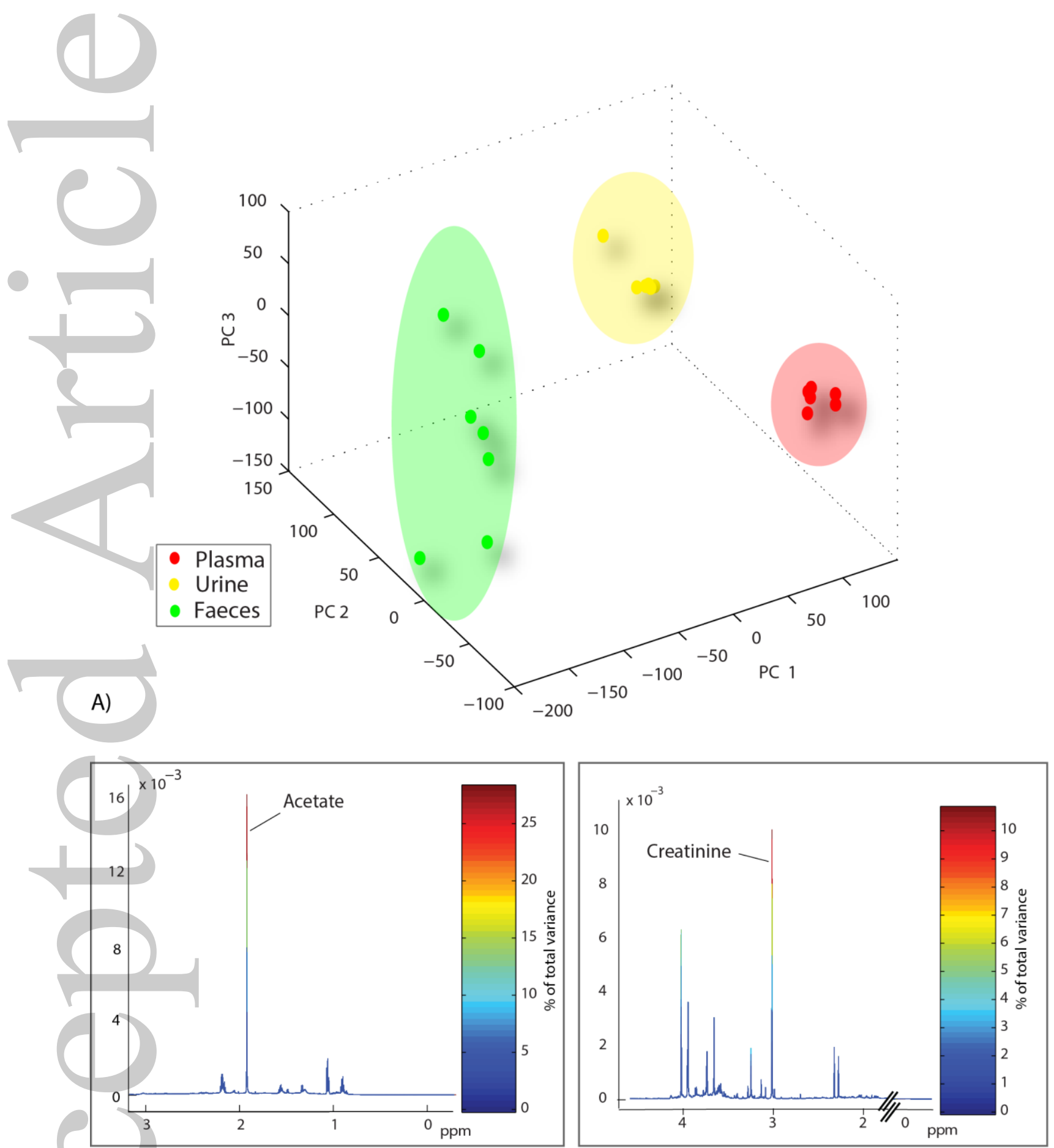

696

697

B)

698
C)

evj_12333_f4 


\begin{tabular}{|c|c|c|c|}
\hline $\mathrm{N}^{\circ}$ & Compound & $\mathrm{N}^{0}$ & Compound \\
\hline 10 & 2-Hydroxy-3-methylbutyric acid & 52 & Isobutyrate \\
\hline 20 & 2-Methylbutyrate & $530 \bigcirc 0$ & Isoleucine \\
\hline 30 & 3-hydroxy-4-methoxymandelic acid & $54 \bigcirc$ & Isovalerate \\
\hline 40 & 3-Hydroxyphenylacetate & $55 \bigcirc \bigcirc 0$ & Lactate \\
\hline 50 & 3-Indoxyl sulphate & $56 \bigcirc \bigcirc$ & Leucine \\
\hline 60 & 3-phenylpropionoate & $57 \bigcirc$ & Lipids \\
\hline 70 & 3-Ureidopropionic acid & 580 & Lysine \\
\hline 80 & 4-hydroxy-3-methoxymandelic acid & $59 \bigcirc$ & Maleic anhydride \\
\hline $9 \bigcirc$ & 5-Aminovalerate & 600 & Malonate \\
\hline 10000 & Acetate & $61 \bigcirc$ & Maltose \\
\hline 1100 & Acetoacetate & 620 & Methionine \\
\hline 120 & Adipate & 630 & Methylguanidine \\
\hline$\frac{12}{12} 0 \Omega$ & $\begin{array}{l}\text { Adipate } \\
\text { Alanine }\end{array}$ & $64 \bigcirc$ & Nicotinurate \\
\hline 13000 & Alanine & $65 \bigcirc$ & Orotate \\
\hline 140 & $\beta$ Alanine & $66 \bigcirc$ & Pantothenate \\
\hline 150 & Allantoate & $67 \bigcirc \bigcirc$ & Phenylacetate \\
\hline 160 & Allantoin & $68 \bigcirc \bigcirc$ & Phenylacetylglycine (PAG) \\
\hline 170 & a-Aminoadipate & $67 \bigcirc$ & Phenylalanine \\
\hline 180 & Beta-Aminoisobutyrate & $69 \bigcirc$ & Proline betaine \\
\hline 190 & Arabinose & 7000 & Phosphocholine \\
\hline 20000 & Arginine & $71 \bigcirc$ & Proline betaine \\
\hline 2100 & Asparagine & 720 & Propionate \\
\hline 220 & Aspartate & 730 & Putrescine \\
\hline 230 & Butyrate & $74 \bigcirc$ & Pyridoxine \\
\hline $24 \bigcirc$ & Caprylate & $75 \bigcirc \bigcirc$ & Pyruvate \\
\hline 2500 & Choline & $76 \bigcirc$ & $p$-cresol glucuronide \\
\hline $26 \bigcirc$ & Cholestrol & $77 \bigcirc$ & $p$-cresol sulphate \\
\hline 2700 & Citrate & $78 \bigcirc \bigcirc$ & p-hydroxybenzoate \\
\hline 2800 & Creatine & $79 \bigcirc 00$ & p-hydroxyphenylacetate \\
\hline $29 \bigcirc \bigcirc$ & Creatinine & 800 & p-hydroxyphenyllactate \\
\hline$\frac{30}{310}$ & Dihydrothymine & $81 \bigcirc$ & p-hydroxyphenylpyruvate \\
\hline 310 & Dimethylamine (DMA) & $82 \bigcirc$ & Quinate \\
\hline$\frac{320}{320}$ & Dimethylglycine (DMG) & $83 \bigcirc$ & Quinone \\
\hline 330 & Dimethyl sulphone & $84 \bigcirc$ & Raffinose \\
\hline 34 & Ethanolamine & $85 \bigcirc \bigcirc$ & Scyllo-inositol \\
\hline$\frac{350}{360} 00$ & Ethyl glucoside & $86 \bigcirc$ & Sebacate \\
\hline 36000 & Formate & $87 \bigcirc \bigcirc$ & Serine \\
\hline$\frac{37}{3800}$ & Glucose & $88 \bigcirc$ & Succinate \\
\hline 38000 & Glutamate & $89 \bigcirc 00$ & Taurine \\
\hline 400 & Glutamine & 90000 & Threonine \\
\hline 41000 & $\begin{array}{l}\text { Glycerol } \\
\text { Glycine }\end{array}$ & $91 \bigcirc 00$ & Trimethylamine- $\mathrm{N}$-oxide (TMAO) \\
\hline 420 & Glycogen & $92 \bigcirc$ & Trans-aconitate \\
\hline 430 & Guanidoacetate & $93 \bigcirc$ & Trigonelline \\
\hline $44 \bigcirc$ & Hippurate & $94 \bigcirc$ & Trimethylamine (TMA) \\
\hline 450 & Histidines & $95 \bigcirc \bigcirc$ & Tyrosine \\
\hline 460 & $\alpha$ Hydroxybutyrate & $96 \bigcirc$ & Uracil \\
\hline 470 & $\beta$ Hydroxybutyrate & $97 \bigcirc$ & Urea \\
\hline $48 \bigcirc \bigcirc$ & Alpha- Hydroxyisobutyrate & 980 & Uridine \\
\hline $49 \bigcirc$ & Hydroquinone & $99 \bigcirc 00$ & Valine \\
\hline 500 & Hypotaurine & $100 \bigcirc$ & Valerate \\
\hline 510 & Indole-3-acetate & $101 \bigcirc$ & Xanthine \\
\hline & & $102 \bigcirc$ & Xylose \\
\hline & ex & $\mathrm{t} 1$ & \\
\hline
\end{tabular}

